\title{
Data Completion Method For the Helmholtz Equation Via Surface Potentials for Partial Cauchy Data
}

\author{
Matthieu Aussal ${ }^{\mathrm{a}}$ Yosra Boukari ${ }^{\mathrm{b} *}$ and Houssem Haddar ${ }^{\mathrm{c}}$ \\ ${ }^{a}$ CMAP, Ecole Polytechnique, \\ Route de Saclay, 91128, Palaiseau Cedex, France \\ b ENIT-LAMSIN, Université de Tunis el-Manar, \\ BP 37, 1002, Tunis, Tunisia \\ c INRIA, CMAP, Ecole Polytechnique, \\ Route de Saclay, 91128, Palaiseau Cedex, France
}

September 23, 2019

\begin{abstract}
We propose and study a data completion algorithm for recovering missing data from the knowledge of Cauchy data on parts of the same boundary. The algorithm is based on surface representation of the solution and is presented for the Helmholtz equation. This work is an extension of the data completion algorithm proposed by the two last authors where the case of data available of a closed boundary was studied. The proposed method is a direct inversion method robust with respect to noisy incompatible data. Classical regularization methods with discrepancy selection principles can be employed and automatically lead to a convergent schemes as the noise level goes to zero. We conduct 3D numerical investigations to validate our method on various synthetic examples.
\end{abstract}

\section{Introduction}

The data completion problem is a basic inverse problem that has been extensively sudied due to its importance as a reference academic problem and in many applications related to electrical non destructive testing. This problem is also naturally encountered in inverse scattering problems where only partial data is available. Since most of the inversion

${ }^{*}$ Corresponding author. Email: yosra.boukari@ensta-b.rnu.tn 
methods are more effective when data are available on a full aperture, the use of a data completion algorithm may be useful in increasing the accuracy of the inversion methods $[13,15]$. We shall study in this paper the use of integral representation theorems to set up a well defined set of equations for the Cauchy problem that can be solved using classical regularization approaches. We discuss the scalar case modeled by the Helmholtz equation at a fixed frequency.

There is a rich literature that deals with the Cauchy problem. A first research stream aims to propose non-iterative methods, such as the work of Lattes and Lions, [20]; Bourgeois $[10,11,12]$, Bourgeois and Dardé,[16]. In this research stream, the authors consider the Laplace case and develop methods to relate missing data to the boundary values of a solution to a stable fourth order problem. Another class of methods focuses on iterative (fix point) schemes. In the case of Laplace problems, the proposed methods use either a Kozlov alternating scheme type (Cimetiere et al. [14]; Ben Belgacem and El Fekih, [6]; Ben Belgacem et al., [8, 7]) or a misfit cost functional minimization (Aboulaich et al.,[1]; Andrieux et al., [3]; Habbal and Kallel,[17]). The former methods have the drawback to be computationally complex and expensive since they require the use of solvers for their numerical implementation, whereas the latter suffer from the issue of not being able to deal with incompatible data, in addition to the high computational cost due to large number of iterations.

It should also be noted that the Helmholtz case was considered by [21] using integral equations and by [5] using the steklov Poincarré formulation, but both of them are iterative methods. We follow here the work in [9] where the authors developed a direct method to solve the Cauchy problem based on integral representation theorems. They considered both the Helmholtz and the Laplace equations but assumed that the boundary can be split in two parts: an exterior boundary and an interior boundary. The proposed method reconstructs the interior Cauchy data by knowing the Cauchy data on the exterior boundary. For their setting, the analysis heavily relies on properties of the Calderon projector associated with a closed boundary. In this paper, we extend the work in [9] by solving the same Cauchy problem under a different boundary specification. We assume that the boundary is split in two non overlapped parts. The difficulty of this case is that the set of integral equations obtained for the inverse problem solution is now posed on open surfaces. Therefore the arguments for the analysis of the method that was employed in [9] are no longer valid. Our analysis in here makes use of lifting functions transforming the problem into a Cauchy problem for screen type problems. We prove that the obtained system has a unique solution. We also prove that the right hand side is always (even for noisy data) in the closure of the range of the operator to invert. The latter property ensures that a selection principle would lead to a convergent scheme. We test our method against synthetic data in 3D configurations. We explain in particular how one can improve the accuracy for Neumann data by solving a direct problem associated with the reconstructed Dirichlet data. We also illustrate how one obtains in practice a (much) higher accuracy for computed interior field using the reconstructed Cauchy data.

The paper is structured as follows. Section 2 presents the setting of the Cauchy problem, the proposed data completion algorithm and we analyze the obtained set of equation to 
prove the convergence for noisy data. In Section 3 we conduct numerical validations on 3D configurations and discuss the accuracy of the proposed algorithm.

\section{Setting of the Cauchy problem and introduction of the data completion algorithm}

Let $\Omega \subset \mathbf{R}^{3}$ be a bounded connected Lipschitz domain and let $\Gamma$ be the boundary of $\Omega$ that can be split into two disjoint parts $\Gamma_{1}$ and $\Gamma_{2}$ that both are open parts with a non zero surface measure. We denote by $\nu$ the normal vector on $\Gamma$ directed to the exterior of $\Omega$ (see Figure 1). Let $u \in H^{1}(\Omega)$ satisfying the following Helmholtz equation inside $\Omega$ :

$$
\Delta u+k^{2} u=0 \quad \text { in } \Omega,
$$

where $k$ denotes the wave number. Our data completion problem consists in determining $\left(u, \partial_{\nu} u\right)_{\mid \Gamma_{2}}$ from the knowledge of $\left(u, \partial_{\nu} u\right)_{\mid \Gamma_{1}}$.

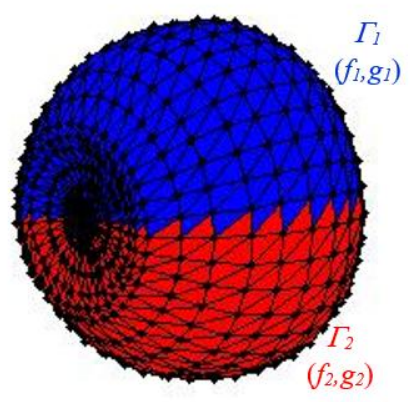

Figure 1: Sketch of the domain

A function $u$ satisfying (1) can be expressed using a surface integral representation as (see for instance [22])

$$
u(x)=S L_{\Gamma}\left(\partial_{\nu} u_{\mid \Gamma}\right)-D L_{\Gamma}\left(u_{\mid \Gamma}\right)
$$

for $x \in \Omega$ where

$$
S L_{\Gamma} \psi(x):=\int_{\Gamma} \psi(y) \Phi(x, y) d s(y), \quad D L_{\Gamma} \varphi(x):=\int_{\Gamma} \varphi(y) \frac{\partial \Phi(x, y)}{\partial \nu(y)} d s(y),
$$


are the single layer and double layer potentials respectively and $\Phi$ the Green function defined for $k \neq 0$ by

$$
\Phi(x, y):=\frac{1}{4 \pi} \frac{e^{i k|x-y|}}{|x-y|} \quad \text { for } \quad x, y \in \mathbf{R}^{3} .
$$

We denote by $(f, g)=\left(u, \partial_{\nu} u\right)_{\mid \Gamma}$ the whole Cauchy data on $\Gamma$ such that

$$
f=\left\{\begin{array}{lll}
f_{1}, & \text { on } & \Gamma_{1} \\
f_{2}, & \text { on } & \Gamma_{2}
\end{array} \quad g=\left\{\begin{array}{lll}
g_{1}, & \text { on } & \Gamma_{1} \\
g_{2}, & \text { on } & \Gamma_{2}
\end{array}\right.\right.
$$

One can see that if $(f, g)$ is known, $u$ can be computed in the entire domain $\Omega$ using (2). Applying trace formulas for layer potentials to (2), one can derive a relation between $\left(f_{1}, g_{1}\right)$, which are the known data, and the unknown data $\left(f_{2}, g_{2}\right)$. These formula will be shown to be sufficient to reconstruct the missing data. This is the scope of next section. Our analysis uses the following classical Sobolev spaces on $\Gamma_{i}(i=1,2)$,

$$
\begin{array}{ll}
H^{1 / 2}\left(\Gamma_{i}\right) & :=\left\{u_{\mid \Gamma_{i}}: \quad u \in H^{1 / 2}(\Gamma)\right\} \\
H^{-1 / 2}\left(\Gamma_{i}\right) & :=\left\{u_{\mid \Gamma_{i}}: \quad u \in H^{-1 / 2}(\Gamma)\right\} \\
\tilde{H}^{1 / 2}\left(\Gamma_{i}\right) & :=\left\{u \in H^{1 / 2}(\Gamma): \quad \operatorname{supp}(u) \subset\left(\Gamma_{i}\right)\right\} \\
\tilde{H}^{-1 / 2}\left(\Gamma_{i}\right) & :=\left\{u \in H^{-1 / 2}(\Gamma): \quad \operatorname{supp}(u) \subset\left(\Gamma_{i}\right)\right\}
\end{array}
$$

We recall that $H^{ \pm 1 / 2}\left(\Gamma_{i}\right)$ are the dual spaces of $\tilde{H}^{\mp 1 / 2}\left(\Gamma_{i}\right), i=1,2$.

\subsection{The data completion Algorithm}

Rewriting (2) as

$$
u(x)=S L_{\Gamma}(g)-D L_{\Gamma}(f) \quad x \in \Omega
$$

and applying the trace and the normal trace formula for single and double layer potentials [22] respectively leads to the following relations

$$
\begin{aligned}
f & =S_{\Gamma}(g)-\left(K_{\Gamma}-\frac{I}{2}\right)(f) \\
g & =\left(K_{\Gamma}^{\prime}+\frac{I}{2}\right)(g)-T_{\Gamma}(f)
\end{aligned}
$$

where the boundary integral operators

$$
\begin{aligned}
S_{\Gamma}: H^{-1 / 2}(\Gamma) \rightarrow H^{1 / 2}(\Gamma), & K_{\Gamma}: H^{1 / 2}(\Gamma) \rightarrow H^{1 / 2}(\Gamma), \\
K_{\Gamma}^{\prime}: H^{-1 / 2}(\Gamma) \rightarrow H^{-1 / 2}(\Gamma), & T_{\Gamma_{i}}: H^{1 / 2}(\Gamma) \rightarrow H^{-1 / 2}(\Gamma),
\end{aligned}
$$

are defined for regular densities $\psi$ and $\varphi$ by:

$$
\begin{array}{rr}
S_{\Gamma} \psi(x):=\int_{\Gamma} \psi(y) \Phi(x, y) d s(y), & K_{\Gamma} \varphi(x):=\int_{\Gamma} \varphi(y) \frac{\partial \Phi(x, y)}{\partial \nu(y)} d s(y), \\
K_{\Gamma}^{\prime} \psi(x):=\int_{\Gamma} \psi(y) \frac{\partial \Phi(x, y)}{\partial \nu(x)} d s(y), & T_{\Gamma} \varphi(x):=\lim _{\epsilon \rightarrow 0} \frac{\partial}{\partial \nu(x)} \int_{\Gamma,|x-y|>\epsilon} \varphi(y) \frac{\partial \Phi(x, y)}{\partial \nu(y)} d s(y) .
\end{array}
$$


One can synthetically rewrite (5)-(6) as $\left(P_{\Gamma}-I\right)(f, g)^{t}=0$ and obtain the classical characterization of the Cauchy data as being in the image of the Calderon projector $P_{\Gamma}: H^{1 / 2}(\Gamma) \times H^{-1 / 2}(\Gamma) \longrightarrow H^{1 / 2}(\Gamma) \times H^{-1 / 2}(\Gamma)$ defined by:

$$
P_{\Gamma}:=\left[\begin{array}{ll}
-K_{\Gamma}+\frac{I}{2} & S_{\Gamma} \\
-T_{\Gamma} & K_{\Gamma}^{\prime}+\frac{I}{2}
\end{array}\right]
$$

One can split this system of equations and reformulate it as a problem for the unknown $\left(f_{2}, g_{2}\right) \in H^{1 / 2}\left(\Gamma_{2}\right) \times H^{-1 / 2}\left(\Gamma_{2}\right)$. Doing so, one ends up with a problem defined on $H^{1 / 2}\left(\Gamma_{2}\right) \times H^{-1 / 2}\left(\Gamma_{2}\right)$ which are not the natural space for surface potentials on screens. To overcome this difficulty, we introduce a lifting couple $(\tilde{f}, \tilde{g}) \in H^{1 / 2}(\Gamma) \times H^{-1 / 2}(\Gamma)$ of the known Cauchy data $\left(f_{1}, g_{1}\right)$ that satisfies

$$
\tilde{f}=\left\{\begin{array}{lll}
f_{1}, & \text { on } & \Gamma_{1} \\
\tilde{f}_{2}, & \text { on } & \Gamma_{2}
\end{array} \quad \tilde{g}=\left\{\begin{array}{lll}
g_{1}, & \text { on } & \Gamma_{1} \\
\tilde{g}_{2}, & \text { on } & \Gamma_{2}
\end{array}\right.\right.
$$

We remark that the existence of $(\tilde{f}, \tilde{g})$ is ensured as long as one assumes that the (possibly noisy) data $\left(f_{1}, g_{1}\right)$ is in $H^{1 / 2}\left(\Gamma_{1}\right) \times H^{-1 / 2}\left(\Gamma_{1}\right)$. We now consider the unknown of our inverse problem as the couple $(\hat{f}, \hat{g}) \in H^{1 / 2}(\Gamma) \times H^{-1 / 2}(\Gamma)$ defined by $\hat{f}=f-\tilde{f}$ and $\hat{g}=g-\tilde{g}$. We observe that

$$
\hat{f}=\left\{\begin{array}{lll}
0 & \text { on } & \Gamma_{1} \\
f_{2}-\tilde{f}_{2} & \text { on } & \Gamma_{2}
\end{array} \text { and } \hat{g}=\left\{\begin{array}{lll}
0 & \text { on } & \Gamma_{1} \\
g_{2}-\tilde{g}_{2} & \text { on } & \Gamma_{2} .
\end{array}\right.\right.
$$

Consequently one can consider the unknown $(\hat{f}, \hat{g})$ of our inverse problem as an element of $\tilde{H}^{1 / 2}\left(\Gamma_{2}\right) \times \tilde{H}^{-1 / 2}\left(\Gamma_{2}\right)$. Since $f=\hat{f}+\tilde{f}$ and $g=\hat{g}+\tilde{g}$, we can rewrite (5)-(6) as an equation for the unknown $(\hat{f}, \hat{g})$ in the form

$$
\left(I-P_{\Gamma}\right)(\hat{f}, \hat{g})^{t}=\left(P_{\Gamma}-I\right)(\tilde{f}, \tilde{g})^{t} .
$$

We recall that the operator $I-P_{\Gamma}$ is not injective. We shall prove that it is injective if restricted to the space $\tilde{H}^{1 / 2}\left(\Gamma_{2}\right) \times \tilde{H}^{-1 / 2}\left(\Gamma_{2}\right)$. We shall also prove that this restriction gives rise to a dense parametrization of the range of $I-P_{\Gamma}$. To avoid the confusion between the operator $I-P_{\Gamma}$ and the operator that we should invert to solve the inverse problem, let us introduce the operator $T$ :

$$
\begin{aligned}
T: \tilde{H}^{1 / 2}\left(\Gamma_{2}\right) \times \tilde{H}^{-1 / 2}\left(\Gamma_{2}\right) & \longrightarrow H^{1 / 2}(\Gamma) \times H^{-1 / 2}(\Gamma) \\
(\varphi, \psi) & \longmapsto\left(I-P_{\Gamma}\right)(\tilde{\varphi}, \tilde{\psi})^{t}
\end{aligned}
$$

where $\tilde{\varphi}$ and $\tilde{\psi}$ denote the extension by zero of $\varphi$ and $\psi$ to the whole boundary $\Gamma$. The inverse problem can be rephrased now as seeking for $(\hat{f}, \hat{g}) \in \tilde{H}^{1 / 2}\left(\Gamma_{2}\right) \times \tilde{H}^{-1 / 2}\left(\Gamma_{2}\right)$ such that

$$
T(\hat{f}, \hat{g})=\left(P_{\Gamma}-I\right)(\tilde{f}, \tilde{g})^{t} .
$$




\subsection{Analysis of (12)}

Our aim is to prove the uniqueness of the solutions to (12) and that the right hand side of (12) is always in the closure of the range of the operator $T$. These two properties are a consequence of the following two lemma.

Lemma 1. For all $\left(v_{1}, v_{2}\right)$ in the range of the operator $\left(P_{\Gamma}-I\right)$ there exists a sequence $\left(\varphi_{n}, \psi_{n}\right)_{n \in \mathbf{N}}$ in $\tilde{H}^{1 / 2}\left(\Gamma_{2}\right) \times \tilde{H}^{-1 / 2}\left(\Gamma_{2}\right)$ such that:

$$
T\left(\varphi_{n}, \psi_{n}\right) \underset{n \rightarrow+\infty}{\longrightarrow}\left(v_{1}, v_{2}\right) \quad \text { in } \quad H^{1 / 2}(\Gamma) \times H^{-1 / 2}(\Gamma)
$$

Proof. To prove this result, it is sufficient to prove that the transpose of $T$ with respect to the duality product

$$
\left\langle(\varphi, \psi),\left(\varphi^{\prime}, \psi^{\prime}\right)\right\rangle=\int_{\Gamma}\left(\varphi \psi^{\prime}+\psi \varphi^{\prime}\right), \quad \forall \varphi, \varphi^{\prime}, \psi, \psi^{\prime} \in L^{2}(\Gamma)
$$

is one to one on the range of $\left(P_{\Gamma}-I\right)$. Since the operators $T_{\Gamma}$ and $S_{\Gamma}$ are symmetric and since $K_{\Gamma}^{\prime}$ is the transpose of $K_{\Gamma}$, one easily concludes that the transpose of $T$ is the operator

$$
\begin{aligned}
T^{\prime}: H^{-1 / 2}(\Gamma) \times H^{1 / 2}(\Gamma) & \rightarrow H^{-1 / 2}\left(\Gamma_{2}\right) \times H^{1 / 2}\left(\Gamma_{2}\right) \\
\left(\varphi^{\prime}, \psi^{\prime}\right) & \left.\mapsto\left(I-P_{\Gamma}^{*}\right)\left(\varphi^{\prime}, \psi^{\prime}\right)^{t}\right|_{\Gamma_{2}}
\end{aligned}
$$

with $P_{\Gamma}^{\prime}$ is the transpose of the Calderon projector given by:

$$
P_{\Gamma}^{\prime}:=\left[\begin{array}{ll}
-K_{\Gamma}^{\prime}+\frac{I}{2} & -T_{\Gamma} \\
S_{\Gamma} & K_{\Gamma}+\frac{I}{2}
\end{array}\right]
$$

Let $\left(v_{1}, v_{2}\right)$ be an element of the range of $\left(P_{\Gamma}-I\right)$ such that:

$$
T^{\prime}\left(v_{1}, v_{2}\right)=0
$$

Using the fact that $P_{\Gamma}$ is a projector $P_{\Gamma}^{2}=P_{\Gamma}$ we deduce that $P_{\Gamma}\left(v_{1}, v_{2}\right)=0$. Then $\left(v_{1}, v_{2}\right) \in H^{1 / 2}(\Gamma) \times H^{-1 / 2}(\Gamma)$ satisfy the following system:

$$
\left\{\begin{array}{lllll}
\left(-K_{\Gamma}+\frac{I}{2}\right)\left(v_{1}\right)+S_{\Gamma}\left(v_{2}\right) & =0 & \text { on } & \Gamma_{2} & (a) \\
-T_{\Gamma}\left(v_{1}\right)+\left(K_{\Gamma}^{\prime}+\frac{I}{2}\right)\left(v_{2}\right) & =0 & \text { on } & \Gamma_{2} & (b) \\
\left(K_{\Gamma}^{\prime}+\frac{I}{2}\right)\left(v_{2}\right)+T_{\Gamma}\left(v_{1}\right) & =0 & \text { on } & \Gamma & (c) \\
-S_{\Gamma}\left(v_{2}\right)-\left(K_{\Gamma}-\frac{I}{2}\right)\left(v_{1}\right) & =0 & \text { on } & \Gamma & (d)
\end{array}\right.
$$

We first observe that

$$
\begin{array}{llll}
(a)-(d) \Rightarrow & S_{\Gamma}\left(v_{2}\right)=0 & \text { on } & \Gamma_{2} \\
(b)-(c) \Rightarrow & T_{\Gamma}\left(v_{1}\right)=0 & \text { on } & \Gamma_{2} .
\end{array}
$$


Let us consider the two functions $w:=S L_{\Gamma}\left(v_{2}\right)$ and $z:=D L_{\Gamma}\left(v_{1}\right)$ which both satisfy the Helmholtz equation outside of $\Gamma$. The last two equality and continuity properties of single and double layer potentials show that

$$
w=0 \quad \text { and } \quad \partial_{\nu} z=0 \quad \text { on } \quad \Gamma_{2} .
$$

On the other hand, we also observe that

$$
\begin{array}{ccccc}
(b)+(c) & \Rightarrow & \left(K_{\Gamma}^{\prime}+\frac{I}{2}\right)\left(v_{2}\right)=0 & \text { on } & \Gamma_{2} \\
(a)+(d) & \Rightarrow & \left(-K_{\Gamma}+\frac{I}{2}\right)\left(v_{1}\right)=0 & \text { on } & \Gamma_{2}
\end{array}
$$

Using (16) and (17) and the trace formulas for layer potentials we respectively conclude that

$$
\partial_{\nu} w^{-}=0 \quad \text { and } \quad z^{-}=0 \quad \text { on } \quad \Gamma_{2}
$$

where the - exponent means that the trace is taken from the interior of the domain $\Omega$. Using (18) and (15) we see that the Cauchy data of $\left.w\right|_{\Omega}$ and $\left.z\right|_{\Omega}$ vanish on $\Gamma_{2}$. Since the two functions satisfy the Helmholtz equation inside $\Omega$ we deduce that

$$
w=0 \quad \text { and } \quad z=0 \quad \text { in } \Omega \text {. }
$$

This implies in particular that

$$
w=0 \quad \text { and } \quad \partial_{\nu} z=0 \text { on } \Gamma \text {. }
$$

We now use the fact that $w$ (respectively $z$ ) satisfies the Helmholtz equation outside $\Omega$ and the Sommerfeld radiation condition. Therefore it is a solution to the homogeneous Dirichlet (respectively Neumann) scattering problem. Consequently

$$
w=0 \quad \text { and } \quad z=0 \quad \text { in } \quad \mathbf{R}^{3} \backslash \Omega
$$

by the uniqueness of solutions to these problems. Using jump relations for the normal trace of single layer potentials and the trace of double layer potentials we arrive at

$$
v_{2}=\left[\partial_{\nu} w\right]=0 \quad \text { and } \quad v_{1}=[z]=0 \quad \text { on } \quad \Gamma
$$

which proves the injectivity of $T^{*}$ and concludes the proof.

Lemma 2. The operator $T: \tilde{H}^{1 / 2}\left(\Gamma_{2}\right) \times \tilde{H}^{-1 / 2}\left(\Gamma_{2}\right) \longrightarrow H^{1 / 2}(\Gamma) \times H^{-1 / 2}(\Gamma)$ is one to one. Proof. Let $(\hat{f}, \hat{g}) \in \tilde{H}^{1 / 2}\left(\Gamma_{2}\right) \times \tilde{H}^{-1 / 2}\left(\Gamma_{2}\right)$ such that:

$$
T(\hat{f}, \hat{g})=0 .
$$

Keeping the same notation for the extension of $(\hat{f}, \hat{g})$ by zero outside $\Gamma_{2}$ we then have

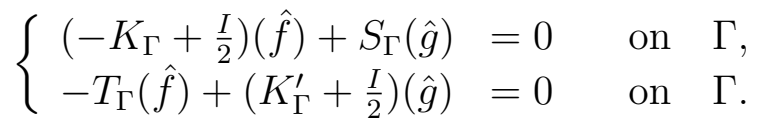


Let us introduce the function $u$ as

$$
u=S L_{\Gamma}(\hat{g})-D L_{\Gamma}(\hat{f}) .
$$

Then, since $(\hat{f}, \hat{g})$ are zero outside $\Gamma_{2}$,

$$
\Delta u+k^{2} u=0 \quad \text { in } \quad \mathbf{R}^{3} \backslash \Gamma_{2} .
$$

Moreover, the restrictions of the equations in (21) to $\Gamma_{1}$ respectively imply

$$
u=0 \quad \text { and } \quad \partial_{\nu} u=0 \text { on } \Gamma_{1} .
$$

Consequently by the unique continuation principle, we obtain that $u$ vanishes in a neighborhood of $\Gamma_{1}$ and consequently in $\mathbf{R}^{3} \backslash \Gamma_{2}$. Then, using the jump relations for the normal trace of single layer potentials and the trace of double layer potentials, we arrive at

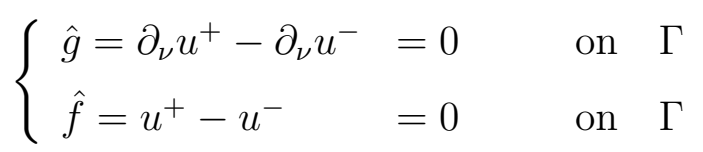

which proves the desired result.

The last two lemma are then sufficient to ensure that a regularization scheme associated with solving (12) for noisy data is convergent if a discrepancy selection principle is applied. We explicit this fact in the following theorem for the Tikhonov regularization associated with the Morozov discrepancy principle. For the proof of this theorem (using the results of the previous Lemmas) we refer for instance to [19]. To simplify the notation we define the operator $B$ by:

$$
\begin{aligned}
B: H^{1 / 2}(\Gamma) \times H^{-1 / 2}(\Gamma) & \longrightarrow H^{1 / 2}(\Gamma) \times H^{-1 / 2}(\Gamma) \\
(\varphi, \psi) & \longmapsto\left(P_{\Gamma}-I\right)(\varphi, \psi)^{t}
\end{aligned}
$$

so that (12) can be witten as

$$
T(\hat{f}, \hat{g})=B(\tilde{f}, \tilde{g}) .
$$

Let us also denote by $X\left(\Gamma_{i}\right):=\tilde{H}^{1 / 2}\left(\Gamma_{i}\right) \times \tilde{H}^{-1 / 2}\left(\Gamma_{i}\right)$.

Corollary 3. Let $(\hat{f}, \hat{g}) \in X\left(\Gamma_{2}\right)$ and $(\tilde{f}, \tilde{g})$ be satisfying $(23)$ and let $\left(\tilde{f}^{\delta}, \tilde{g}^{\delta}\right) \in X(\Gamma)$ be such that

$$
\left\|\left(\tilde{f}^{\delta}, \tilde{g}^{\delta}\right)-(\tilde{f}, \tilde{g})\right\|_{X(\Gamma)}<\delta .
$$

Consider the Tikhonov solution $\left(\hat{f}^{\delta}, \hat{g}^{\delta}\right)$ satisfying

$$
\left(\alpha(\delta) I+T^{*} T\right)\left(\hat{f}^{\delta}, \hat{g}^{\delta}\right)=T^{*} B\left(\tilde{f}^{\delta}, \tilde{g}^{\delta}\right)
$$

where $\alpha(\delta)$ is determined (for sufficiently small $\delta$ ) using the Morozov discrepancy principle, i.e $\alpha(\delta)$ is the unique solution of

$$
\left\|T\left(\hat{f}^{\delta}, \hat{g}^{\delta}\right)-B\right\|_{X(\Gamma)}=\delta\|B\| .
$$

Then

$$
\left\|\left(\hat{f}^{\delta}, \hat{g}^{\delta}\right)-(\hat{f}, \hat{g})\right\|_{X\left(\Gamma_{2}\right)} \longrightarrow 0 \quad \text { as } \quad \delta \longrightarrow 0 .
$$




\section{Numerical scheme and validation}

The goal of this section is to test the accuracy and robustness of the numerical inversion procedure described in Corollary (3) for 3D synthetic configurations. In order to build the matrices associated with the surface operators $T$ and $B$ we rely on Gipsylab [2][4] library. For a given surface $\Gamma$, we use a triangulation as in the example of Figure 1. Writing variationally the equality (23) we discretize the obtained system of equations using piecewise linear finite element basis. If $N$ denotes the number of vertices of the surface mesh and $M$ the number of vertices inside $\Gamma_{2}$, then the discretization of the operator $T$ leads to a matrix $\mathbb{T} \in \mathbf{C}^{2 N \times 2 M}$ and the discretization of the operator $B$ leads to a matrix $\mathbb{B} \in \mathbf{C}^{2 N \times 2 N}$. The solution of the inverse problem is then obtained by solving (24) where $T$ and $B$ are respectively replaced by $\mathbb{T}$ and $\mathbb{B}$. As explained in the analysis of the method, an extension operator is needed to build $\tilde{f}$ and $\tilde{g}$. For our numerical examples, we used the simple extension by 0 operator. Although it may be considered as the worst possible extension operator from the numerical point of view (since the continuity modulus of this extension operator would explode as the mesh size goes to zero), we observed that it numerically yields reasonable accuracy for the inverse problem.

In order to simulate the synthetic data $\left(f_{1}, g_{1}\right)$ and use a quite arbitrary set of data, we choose to solve a scattering problem for an inclusion $\tilde{\Omega}$ (which is taken to be a sphere in our examples) modeled with Neumann boundary conditions such that the domain $\Omega \subset \mathbf{R}^{3} \backslash \tilde{\Omega}$. This way, the numerical data is constructed using none of the matrix blocs of $\mathbb{T}$ and $\mathbb{B}$.

In the following tests $\tilde{\Omega}$ is the sphere with center $(1.5,1.5,1.5)$ and with radius 0.5 and we use a plane wave as incident wave $e^{i k d \cdot x}$ where the unitary direction $d$ will be specified for each example. To model measurements noise in the data, we corrupt the synthetic data $\left(f_{2}, g_{2}\right)$ with random noise of level $\delta$ and we compute the noisy data as

$$
\left(f_{2}^{\delta}, g_{2}^{\delta}\right)=\left(f_{2}^{\delta}, g_{2}^{\delta}\right) \cdot *\left(1+\delta\left(\theta_{1}, \theta_{2}\right)\right)
$$

where $\theta_{1}$ and $\theta_{2}$ have complex entries with imaginary and real parts randomly and uniformly chosen between -1 and 1 and where .* denotes component wise multiplication between vectors.

Example 1. We start with the simple example where the domain $\Omega$ is the unit ball centered at the origin and take as incident plane wave direction $d=(0,0,-1)$. We first analyze the accuracy for different wave numbers in the favorable case where the aperture $\Gamma_{2}=\{(x, y, z) \in \partial \Omega ; x<-0.75\}$ is large compared to the part $\Gamma_{1}$ where the data is missing. The results are shown in Table 1 for three different frequencies $k=0.25, k=1$ and $k=2$. For these frequencies, the dimensions of $\Gamma_{1}$ are less than a wavelength. In order to measure the accuracy we compute the relative $L^{2}\left(\Gamma_{1}\right)$ norm between the reconstructed field and the exact one.

We observe that the reconstruction of the solution $u$ is very accurate and robust with respect to the noise for all frequencies. However, for the Neumann component when the noise becomes relatively large $(5 \%)$ the accuracy is poor. As we will illustrate later, one 
can improve the accuracy on the Neumann part by using the reconstructed trace $u$ on the whole boundary $\partial \Omega$. We shall also illustrate that even if the reconstructed Neumann data may not be accurate, the field inside the domain can be accurately computed using the integral representation formula (2).

\begin{tabular}{|c|c|c|c|c|c|c|c|}
\hline \multicolumn{4}{|c|}{$k=0.25$} & \multicolumn{4}{|c|}{$k=1$} \\
\hline$\delta$ & $10^{-3}$ & $10^{-2}$ & $5 \quad 10^{-2}$ & $\delta$ & $10^{-3}$ & $10^{-2}$ & $510^{-2}$ \\
\hline $\mathrm{u}$ & 0.009 & 0.03 & 0.03 & $\mathrm{u}$ & 0.001 & 0.007 & 0.03 \\
\hline$\partial_{\nu} u$ & 0.02 & 0.08 & 0.2 & $\partial_{\nu} u$ & 0.01 & 0.06 & 0.2 \\
\hline \multicolumn{6}{|c|}{$k=2$} & & \\
\hline & & $\delta$ & $10^{-3}$ & $10^{-2}$ & $5 \quad 10^{-2}$ & & \\
\hline & & $\mathrm{u}$ & 0.01 & 0.03 & 0.03 & & \\
\hline & & $\partial_{\nu} u$ & 0.05 & 0.06 & 0.19 & & \\
\hline
\end{tabular}

Table 1: Relative error in $L^{2}\left(\Gamma_{1}\right)$ of the reconstructed Cauchy data associated with Example 1 and for three different wave numbers $k$.

For the same configuration of Example 1, we now test the efficiency of our algorithm with respect to size of $\Gamma_{1}$ for a fixed wave number $k=0.5$. We define the domain $\Gamma_{1}=$ $\left\{(x, y, z) \in \partial \Omega ; x<x_{0}\right\}$ and we increase the size of $\Gamma_{1}$ by increasing the value of $x_{0}$. Table 2 illustrates the obtained results for $x_{0}=-0.75$, 0 , and 0.25 respectively. We indeed observe that the quality of the results decreases as the size of $\Gamma_{1}$ increases, especially for the normal derivative. The accuracy for the reconstruction of the field trace remains very high.
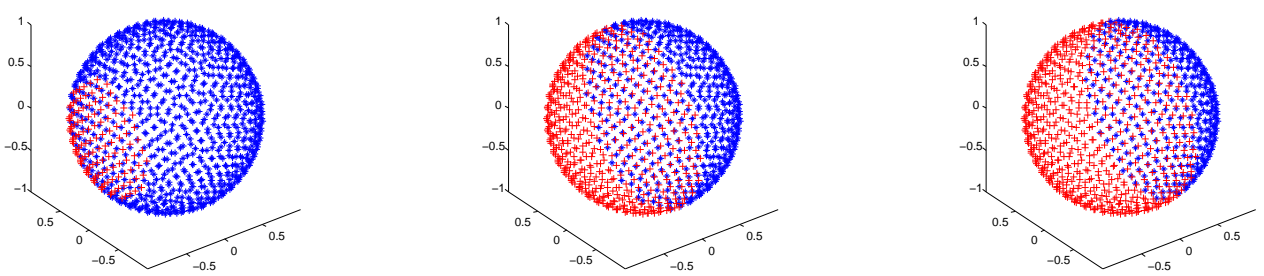

Figure 2: Setting of the tested domains $\Gamma_{1}$ in red and $\Gamma_{2}$ in blue. $\Gamma_{1}=$ $\left\{(x, y, z) \in \partial \Omega ; x<x_{0}\right\}, x_{0}=-0.75$ (left), $x_{0}=0$ (middle) and $x_{0}=0.25$ (right). 


\begin{tabular}{|c|c|c|c|c|c|c|c|c|c|c|c|}
\hline \multicolumn{4}{|c|}{$x_{0}=-0.75$} & \multicolumn{4}{|c|}{$x_{0}=0$} & \multicolumn{4}{|c|}{$x_{0}=0.25$} \\
\hline$\delta$ & $10^{-3}$ & $10^{-2}$ & $510^{-2}$ & $\delta$ & $10^{-3}$ & $10^{-2}$ & $510^{-2}$ & $\delta$ & $10^{-3}$ & $10^{-2}$ & $510^{-2}$ \\
\hline $\mathrm{u}$ & 0.01 & 0.02 & 0.03 & $\mathrm{u}$ & 0.02 & 0.05 & 0.06 & $u$ & 0.02 & 0.07 & 0.09 \\
\hline$\partial_{\nu} u$ & 0.09 & 0.07 & 0.28 & $\partial_{\nu} u$ & 0.05 & 0.11 & 0.23 & $\partial_{\nu} u$ & 0.16 & 0.16 & 0.31 \\
\hline
\end{tabular}

Table 2: Relative error in $L^{2}\left(\Gamma_{1}\right)$ for a fixed wavenumber $k=0.5$ and different aperture sizes depicted in Figure 2

Example 2. In a second example we test the case of non smooth geometries by considering the case of a cuboid where data is missing on one of its faces. The example is illustrated in Figure (3) where $\partial \Omega$ is the rectangular cuboid $[-0.5,0.5] \times[-0.5,0.5] \times[-0.25,0.25]$ and the surface $\Gamma_{1}$ occupies the lower face $[-0.5,0.5] \times[-0.5,0.5] \times\{-0.25\}$. The incident plane wave direction is $d=(0,0,-1)$. This test is more challenging since singularities may occur at the edges of the cube and affect the precision of the reconstruction. Figure 3 shows the obtained reconstructions for $k=0.25$ and the added noise level $\delta=0.01$ where a good accuracy is observed. This is confirmed by Table 3 where the relative error in $L^{2}\left(\Gamma_{1}\right)$ is reported for different frequencies and noise levels. We again observe that while a good reconstruction for the trace of the solution is observed, the normal derivative is poorly reconstructed when the noise level is high.
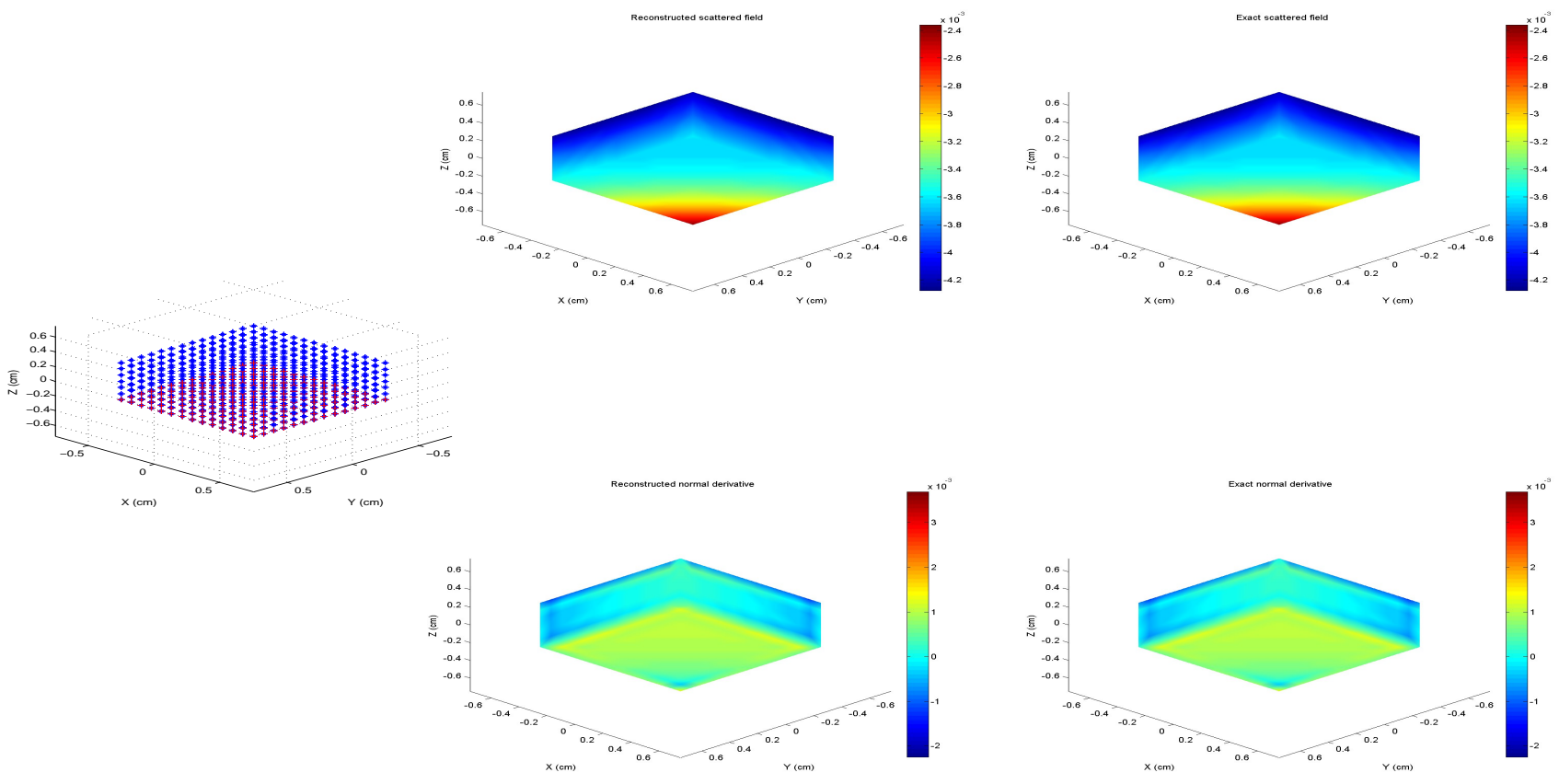

Figure 3: Configuration of $\Gamma_{1}$ (red) and $\Gamma_{2}$ (blue) on the left. The four figures on the right show the exact fields (first column) and reconstructed fields (right column) for the trace (first row) and the normal trace (second row). These results correspond to $k=0.25$, a plane wave with direction $d=(0,0,-1)$ and an added noise $\delta=0.01$. 


\begin{tabular}{|c|c|c|c|c|c|c|c|}
\hline \multicolumn{4}{|c|}{$k=0.25$} & \multicolumn{4}{|c|}{$k=0.5$} \\
\hline$\delta$ & $10^{-3}$ & $10^{-2}$ & $510^{-2}$ & $\delta$ & $10^{-3}$ & $10^{-2}$ & $510^{-2}$ \\
\hline $\mathrm{u}$ & 0.005 & 0.005 & 0.02 & $\mathrm{u}$ & 0.008 & 0.05 & 0.05 \\
\hline$\partial_{\nu} u$ & 0.05 & 0.17 & 0.6 & $\partial_{\nu} u$ & 0.05 & 0.16 & 0.5 \\
\hline & & \multicolumn{4}{|c|}{$k=1$} & & \\
\hline & & $\delta$ & $10^{-3}$ & $10^{-2}$ & $510^{-2}$ & & \\
\hline & & $\mathrm{u}$ & 0.006 & 0.058 & 0.02 & & \\
\hline & & $\partial_{\nu} u$ & 0.046 & 0.13 & 0.5 & & \\
\hline
\end{tabular}

Table 3: Relative $L^{2}\left(\Gamma_{1}\right)$ error for different wave numbers $k$ and noise levels $\delta$ associated with Example 2 and the configuration of $\partial \Omega$ depicted in Figure 3-left: $\partial \Omega=[-0.5,0.5] \times$ $[-0.5,0.5] \times[-0.25,0.25]$ and $\Gamma_{1}=[-0.5,0.5] \times[-0.5,0.5] \times\{-0.25\}$.

Example 3. The following examples aim at showing reconstructions for complex geometries and illustrating the possible achievements of the algorithm for these configurations. Although motivated by applications related to imaging inside the brain, the examples do not correspond to a specific experiment and choosing the shape of a head was mainly motivated by available surface meshes [18] that can be used for building the surface operator matrices associated with our inversion algorithm.

In the first battery of tests, in addition to test the accuracy of the algorithm for different frequencies and noise levels, we also show how the results for the normal derivative may be slightly improved using the obtained trace on the whole boundary. The difference in accuracy between the trace and normal trace may come from the fact that the regularization parameter is not optimal for both components. Therefore, it may be interesting to use the reconstructed trace on $\partial \Omega$ (for which we observed a much higher accuracy) to solve a Dirichlet problem for the Helmholtz equation inside $\Omega$ then compute the normal derivative from the computed solution. These two steps can be done using integral representation formulas (2). Taking the normal trace in (2) we obtain

$$
\left(K_{\Gamma}^{\prime}+I\right) \partial_{\nu} u_{\Gamma}=T_{\Gamma} u_{\Gamma}
$$

Therefore, if a reconstructed version of $u_{\Gamma}$ is obtained, say $u_{\Gamma}^{\delta}$, we compute $\partial_{\nu} u_{\Gamma}^{\delta}$ through

$$
\partial_{\nu} u_{\Gamma}^{\delta}=\left(K_{\Gamma}^{\prime}+I\right)^{-1} T_{\Gamma} u_{\Gamma}^{\delta}
$$

where $\left(K_{\Gamma}^{\prime}+I\right)^{-1}$ exists if $k^{2}$ is not a Neumann eigenvalue for the Laplace operator inside $\Omega[22]$. The continuity of the operator $\left(K_{\Gamma}^{\prime}+I\right)^{-1} T_{\Gamma}$ from $H^{1 / 2}(\Gamma) \rightarrow H^{-1 / 2}(\Gamma)$ then guarantees that the error amplification on $\partial_{\nu} u_{\Gamma}^{\delta}$ would be linear in terms of the error on $u_{\Gamma}^{\delta}$ measured in $H^{1 / 2}(\Gamma)$. Controlling the $H^{1 / 2}(\Gamma)$ error would require a smoothing of the reconstructed field $u_{\Gamma}^{\delta}$. In the following examples this step is skipped and we apply (28) directly to the obtained $u_{\Gamma}^{\delta}$ from the inversion algorithm. 


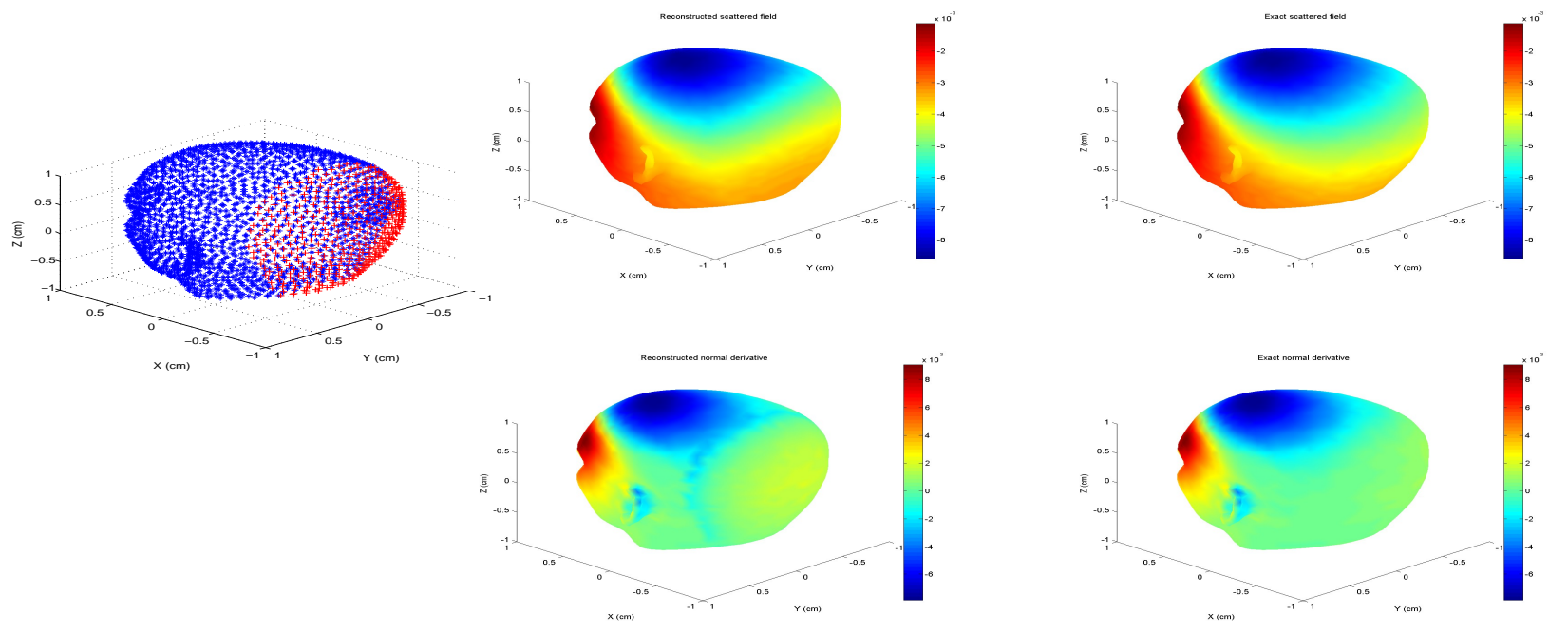

Figure 4: Configuration of $\Gamma_{1}$ (red) and $\Gamma_{2}$ (blue) on the left. The four figures on the right show the exact fields (first column) and reconstructed fields (right column) for the trace (first row) and the normal trace (second row). These results correspond to $k=0.25$, a plane wave with direction $d=(0,0,-1)$ and an added noise $\delta=0.01$.

\begin{tabular}{|l|c|c|c|}
\hline \multicolumn{4}{|c|}{$k=0.25$} \\
\hline$\delta$ & $10^{-3}$ & $10^{-2}$ & $510^{-2}$ \\
\hline $\mathrm{u}$ & 0.002 & 0.01 & 0.03 \\
$\partial_{\nu} u$ & 0.02 & 0.082 & 0.4 \\
\hline
\end{tabular}

\begin{tabular}{|l|c|c|c|}
\hline \multicolumn{4}{|c|}{$k=1$} \\
\hline$\delta$ & $10^{-3}$ & $10^{-2}$ & $510^{-2}$ \\
\hline $\mathrm{u}$ & 0.006 & 0.011 & 0.04 \\
$\partial_{\nu} u$ & 0.05 & 0.09 & 0.4 \\
\hline
\end{tabular}

\begin{tabular}{|l|c|c|c|}
\hline \multicolumn{4}{|c|}{$k=2$} \\
\hline$\delta$ & $10^{-3}$ & $10^{-2}$ & $510^{-2}$ \\
\hline $\mathrm{u}$ & 0.0096 & 0.02 & 0.04 \\
$\partial_{\nu} u$ & 0.06 & 0.1 & 0.3 \\
\hline
\end{tabular}

Table 4: Relative $L^{2}\left(\Gamma_{1}\right)$ error for different wave numbers $k$ and noise levels $\delta$ associated with Example 3 (see Figure 4-left). The Neumann data is computed using (28).

In order to see the improvement provided by the post-processing provided by (28), we provide in Table 5 the relative error for the normal derivative that was obtained without post-processing. While the relative error remains important for a noise level of the order of $5 \%$, we observe a good improvement for lower noise levels. 


\begin{tabular}{|l|c|c|c|}
\hline$\delta$ & $10^{-3}$ & $10^{-2}$ & $510^{-2}$ \\
\hline \hline$k=0.25$ & 0.2 & 0.3 & 0.43 \\
$k=1$ & 0.22 & 0.2 & 0.41 \\
$k=2$ & 0.2 & 0.28 & 0.4 \\
\hline
\end{tabular}

Table 5: Relative error for the normal derivative associated with Example 3 (see Figure 4left) without post-processing (28) for different wave numbers $k$ and noise levels $\delta$. Compare with Table 4.

Example 4. We illustrate in this example how a good accuracy is achieved for the reconstruction of the field in the interior of $\Omega$ even though the normal derivative is not well reconstructed at the interface between $\Gamma_{1}$ and $\Gamma_{2}$. In the example we assume that the domain $\Omega$ is a ball of radius 1 and the data is available on $\Gamma_{2}=\{(x, y, z) \in \partial \Omega ; x>-0.5\}$. Whence the Cauchy data is reconstructed on $\Gamma_{1}$ leading to a knowledge of $u_{\Gamma}^{\delta}$ and $\partial_{\nu} u_{\Gamma}^{\delta}$, we evaluate the field and the normal derivative on the inner surface using the integral representation (2). We observe that the accuracy obtained on the inner surface is very good as shown in Table 6 . The error is much lower than the one obtained on $\partial \Omega$. This is explained by the fact that the error contained in $\partial_{\nu} u_{\Gamma}^{\delta}$ is mainly due to high oscillations. Since the single and double layer potentials are smoothing operators when evaluated at a surface not intersecting $\partial \Omega$, the error contained in $\partial_{\nu} u_{\Gamma}^{\delta}$ is smoothed out and the accuracy of the reconstructed field appears better.

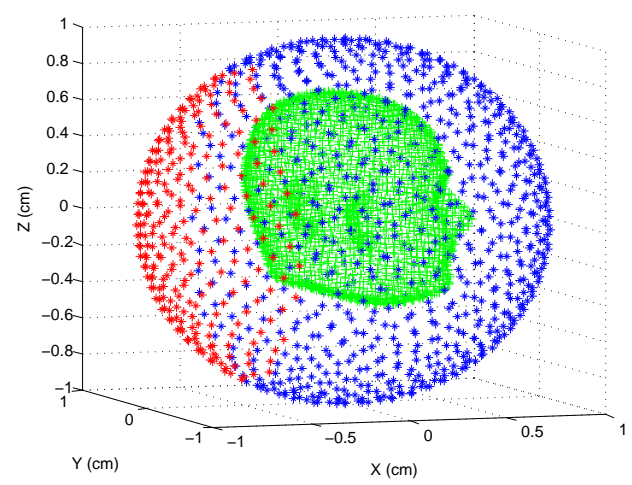

Figure 5: Configuration of $\Gamma_{1}$ (red) and $\Gamma_{2}$ (blue) on the sphere. The mesh of the head is a fictitious surface where the field will be evaluated. 

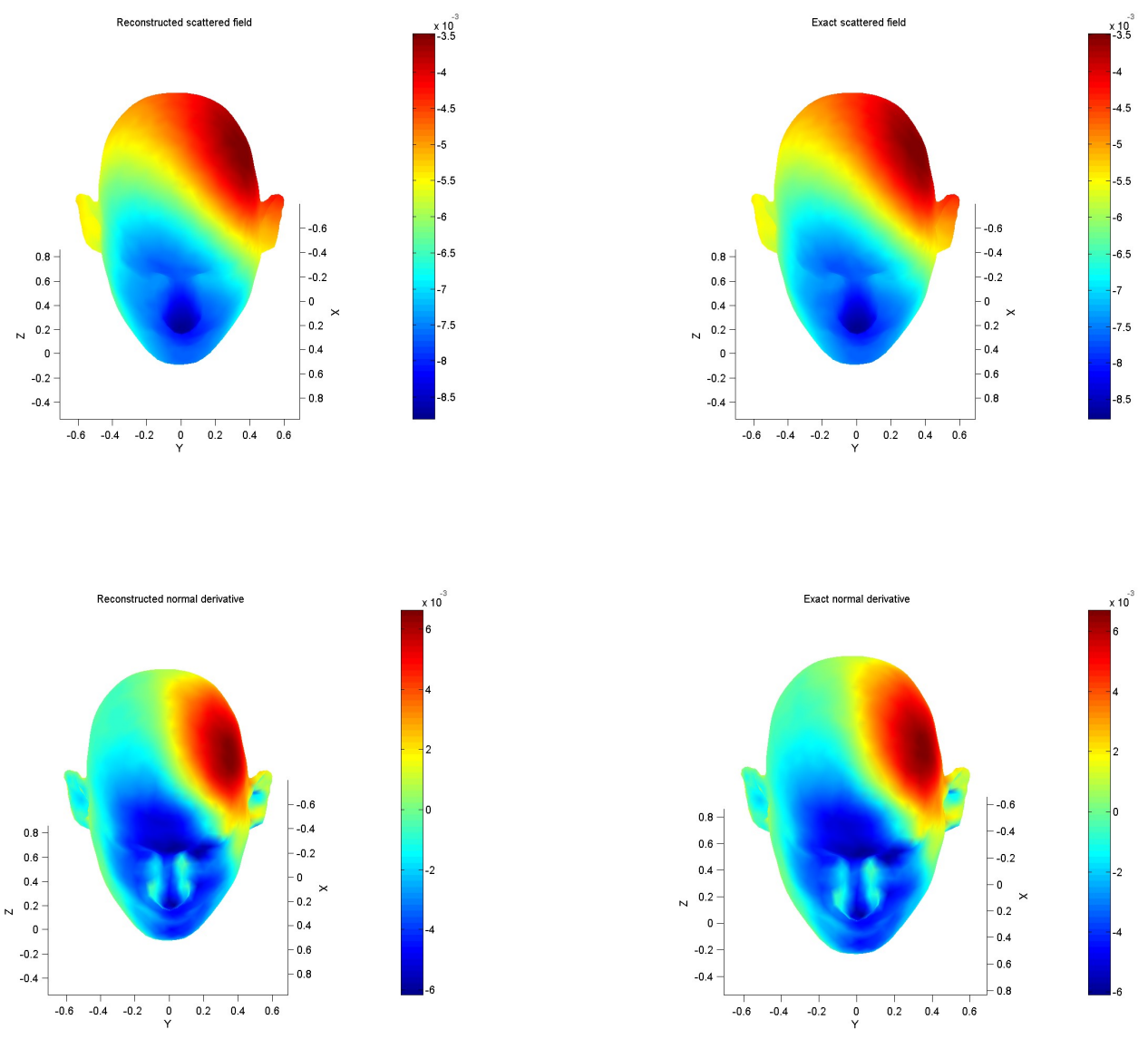

Figure 6: Results associated with Example 4. The four figures show the exact fields (first column) and reconstructed fields (right column) for the trace (first row) and the normal trace (second row). These results correspond to $k=0.5$, a plane wave with direction $d=(0,0,-1)$ and an added noise $\delta=0.01$. 


\begin{tabular}{|l|c|c|c||l|c|c|c|}
\hline \multicolumn{5}{|c|}{$k=0.25$} & \multicolumn{4}{|c|}{$k=1$} \\
\hline$\delta$ & $10^{-3}$ & $10^{-2}$ & $510^{-2}$ & $\delta$ & $10^{-3}$ & $10^{-2}$ & $510^{-2}$ \\
\hline $\mathrm{u}$ & 0.001 & 0.003 & 0.01 & $\mathrm{u}$ & 0.0004 & 0.006 & 0.02 \\
$\partial_{\nu} u$ & 0.008 & 0.01 & 0.05 & $\partial_{\nu} u$ & 0.001 & 0.008 & 0.027 \\
\hline
\end{tabular}

Table 6: Results associated with Example 4. Relative $L^{2}$ error for the field reconstructed on an inner surface of the domain $\Omega$ for different wave numbers $k$ and noise levels $\delta$. The configuration of the domain is explained in Figure 5.

\begin{tabular}{|l|c|c|c|}
\hline \multicolumn{4}{|c|}{$k=0.25$} \\
\hline$\delta$ & $10^{-3}$ & $10^{-2}$ & $510^{-2}$ \\
\hline $\mathrm{u}$ & 0.005 & 0.01 & 0.1 \\
$\partial_{\nu} u$ & 0.02 & 0.1 & 0.3 \\
\hline
\end{tabular}

\begin{tabular}{|l|c|c|c|}
\hline \multicolumn{4}{|c|}{$k=1$} \\
\hline$\delta$ & $10^{-3}$ & $10^{-2}$ & $510^{-2}$ \\
\hline$u$ & 0.004 & 0.01 & 0.08 \\
$\partial_{\nu} u$ & 0.02 & 0.09 & 0.2 \\
\hline
\end{tabular}

\begin{tabular}{|l|c|c|c|}
\hline \multicolumn{4}{|c|}{$k=2$} \\
\hline$\delta$ & $10^{-3}$ & $10^{-2}$ & $510^{-2}$ \\
\hline$u$ & 0.008 & 0.01 & 0.03 \\
$\partial_{\nu} u$ & 0.02 & 0.08 & 0.2 \\
\hline
\end{tabular}

Table 7: Results associated with Example 4. Relative $L^{2}$ error for the field on $\partial \Omega$ for different wave numbers $k$ and noise levels $\delta$. The configuration of the domain is explained in Figure 5.

\section{Acknowledgement}

The research of Y. BOUKARI was supported by the Tunisian Ministry of higher Education and Research through the research project 18PJEC05-02.

\section{References}

[1] R. Aboulaïch, A. Ben Abda, and M. Kallel. Missing boundary data reconstruction via an approximate optimal control. Inverse Probl. Imaging, 2(4):411-426, 2008.

[2] F. Alouges and M. Aussal. Fem and bem simulations with the gypsilab framework. SMAI-JCM, 4:297-318, 2018.

[3] S. Andrieux, T. N. Baranger, and A. Ben Abda. Solving Cauchy problems by minimizing an energy-like functional. Inverse Problems, 22(1):115-133, 2006.

[4] M. Aussal. Gypsilab. http://www.cmapx.polytechnique.fr/ aussal/\# LOGICIELS, started on 2018.

[5] A. Ben Abda, R. Ben Fatma, and D. Tromeur-Dervout. An Aitken-like acceleration method applied to missing boundary data reconstruction for the Cauchy-Helmholtz problem. C. R. Math. Acad. Sci. Paris, 348(1-2):93-97, 2010. 
[6] F. Ben Belgacem. Why is the Cauchy problem severely ill-posed? Inverse Problems, 23(2):823-836, 2007.

[7] F. Ben Belgacem, D. Du, and F. Jelassi. Extended-domain-Lavrentiev's regularization for the Cauchy problem. Inverse Problems, 27(4):045005, 27, 2011.

[8] F. Ben Belgacem and H. El Fekih. On Cauchy's problem. I. A variational SteklovPoincaré theory. Inverse Problems, 21(6):1915-1936, 2005.

[9] Y. Boukari and H Haddar. A convergent data completion algorithm using surface integral equations. Inverse Problems, pages 035-011, 2015.

[10] L. Bourgeois. Convergence rates for the quasi-reversibility method to solve the Cauchy problem for Laplace's equation. Inverse Problems, 22(2):413-430, 2006.

[11] L. Bourgeois. About stability and regularization of ill-posed elliptic Cauchy problems: the case of $C^{1,1}$ domains. M2AN Math. Model. Numer. Anal., 44(4):715-735, 2010.

[12] L. Bourgeois and J. Dardé. A duality-based method of quasi-reversibility to solve the Cauchy problem in the presence of noisy data. Inverse Problems, 26(9):095016, 21, 2010.

[13] F. Cakoni and R. Kress. Integral equations for inverse problems in corrosion detection from partial Cauchy data. Inverse Probl. Imaging, 1(2):229-245, 2007.

[14] A. Cimetière, F. Delvare, M. Jaoua, and F. Pons. Solution of the Cauchy problem using iterated Tikhonov regularization. Inverse Problems, 17(3):553-570, 2001.

[15] D. Colton and R. Kress. Inverse acoustic and eletromagnetic scattering theory. Springer, New York, 2nd edition, 1998.

[16] J. Dardé, A. Hannukainen, and N. Hyvönen. An $H_{d i v}$-based mixed quasi-reversibility method for solving elliptic Cauchy problems. SIAM J. Numer. Anal., 51(4):2123-2148, 2013.

[17] A. Habbal and M. Kallel. Neumann-Dirichlet Nash strategies for the solution of elliptic Cauchy problems. SIAM J. Control Optim., 51(5):4066-4083, 2013.

[18] C. Jin and T. Tew. Morphoacoustics. https://www.morphoacoustics.org/resources.html, started on 2002.

[19] A. Kirsch. An Introduction to the Mathematical Theory of Inverse Problems. Springer, New York, 2nd edition, 2011.

[20] R. Lattès and J.-L. Lions. Méthode de quasi-réversibilité et applications. Travaux et Recherches Mathématiques, No. 15. Dunod, Paris, 1967. 
[21] L. Marin, L. Elliott, P. J. Heggs, D. B. Ingham, D. Lesnic, and X. Wen. Comparison of regularization methods for solving the Cauchy problem associated with the Helmholtz equation. Internat. J. Numer. Methods Engrg., 60(11):1933-1947, 2004.

[22] W. McLean. Strongly Elliptic Systems and Boundary Integral Operators. Cambridge University Press, Cambridge, UK, 2000. 